\title{
Dilemas éticos de las trabajadoras y los trabajadores sociales en España
}

\author{
Alberto Ballestero Izquierdo \\ María Jesús Úriz Pemán \\ Juan Jesús Viscarret Garro \\ Universidad Pública de Navarra. Departamento de Trabajo Social \\ alberto.ballestero@unavarra.es; ivan@unavarra.es; juanj.viscarret@unavarra.es
}

\section{Resumen}

El principal objetivo de este artículo es presentar los principales dilemas éticos con los que se encuentran las trabajadoras y los trabajadores sociales españoles en su ejercicio profesional. Para ello, expondremos parte de los resultados obtenidos a través del proyecto de investigación Dilemas éticos en la intervención social: La perspectiva de los trabajadores sociales en España (FFI2008-05546). Se trata de un proyecto I+D+i desarrollado en España por el grupo EFIMEC (Ética, Filosofía y Metodología de la ciencia) de la Universidad Pública de Navarra financiado por el Ministerio de Ciencia e Innovación. Además, presentamos las principales cuestiones que están en el debate general sobre los dilemas éticos en trabajo social y situamos los hallazgos obtenidos en dicha discusión.

Palabras clave: dilemas éticos; trabajo social; intervención social; autonomía; confidencialidad; competencia profesional; información a terceros.

\section{Abstract. Ethical dilemmas of social workers in Spain}

This article examines the main ethical dilemmas that Spanish social workers face in professional practice and explores the methodologies that they use to overcome such dilemmas. To achieve our aim, we present some of the results obtained in the research project titled "Ethical dilemmas in social intervention. The perspective of Spanish social workers" (FFI2008-05546). This is an R\&D\&I project that is being conducted in Spain by the research group EFIMEC (Ética, Filosofía y Metodología de la ciencia) at the Universidad Pública de Navarra (Spain) and financed by the Spanish Ministry of Science and Innovation. We also examine the general debate about ethical dilemmas in social work and attempt to frame our findings in the context of the discussion.

Keywords: ethical dilemmas; social work; social intervention; autonomy; confidentiality; professional competence; information to third parties. 


\author{
Sumario \\ Introducción Discusión \\ Metodología Conclusiones \\ Resultados Agradecimientos \\ Referencias bibliográficas
}

\title{
Introducción
}

Como señala Preston (2001), la ética es intrínseca y desempeña un papel central en la teoría y en la práctica del trabajo social, así como en el resto de las llamadas "profesiones sociales", es decir, en las profesiones que se ocupan de la atención, el cuidado, la educación y la defensa de las personas o los grupos más vulnerables de nuestras sociedades (Lorenz y Seibel, 1999; Banks, 2004). El trabajo social se ocupa de aspectos fundamentales de la vida de las personas $\mathrm{y}$ de sus necesidades, trata con individuos, familias o grupos que necesitan una intervención apropiada que les ayude a mejorar sus condiciones de vida, su autonomía y su bienestar.

Las cuestiones éticas están profundamente arraigadas en la disciplina de trabajo social, dado que precisamente en la intervención social se visualizan y se concretan los principales dilemas éticos que tienen que resolver estos profesionales. Como muy bien expresan Loewenberg y Dolgoff (1996: XIII): «los problemas éticos surgen cuando y donde la gente tiene que ocuparse de la vida humana, de su supervivencia y su bienestar. En esas actividades, se implican diariamente los trabajadores sociales».

Podría decirse que el interés y la literatura sobre los dilemas éticos en trabajo social ha crecido durante las últimas décadas, sobre todo en el ámbito anglosajón (Rhodes, 1991; Congress, 1999; Linzer, 1999; McAuliffe, 2000). Reamer (1990: IX) señalaba: «[...] el creciente interés en la ética profesional y más concretamente en la ética del trabajo social. Esto refleja una mayor conciencia de la dimensión ética de la práctica y una mayor comprensión del contexto ético en el que a menudo se realizan los juicios profesionales». En España, la preocupación por los aspectos éticos de la profesión de trabajo social también se refleja en varios trabajos (Salcedo, 1998; Bermejo, 2002; Ballestero, 2006).

En general, existe el acuerdo bastante generalizado sobre el hecho de que un dilema aparece cuando hay un conflicto entre principios que conduce a cursos de acción excluyentes (McAuliffe, 2005; Reamer, 1983; Rothman, 1998; Congress, 1999; Banks, 2001). Por ejemplo, podemos decir que hay un dilema ético cuando se da un conflicto entre el derecho de un usuario a ser informado y el cumplimiento de una norma de una organización contraria a ese derecho, o cuando el trabajador o la trabajadora social ha de tomar una decision que implica un conflicto entre los principios de autonomía y de bienestar relativos al usuario. Matizando aún más, se podría decir que un dilema ético supone «una situación de toma de decisiones que conlleva una difícil elección entre 
dos alternativas igualmente no deseables y en la que no está claro qué elección es la correcta» (Banks, 2005: 1011).

Sin duda alguna, identificar los dilemas éticos a los que se enfrentan los trabajadores y las trabajadoras sociales, medir su impacto y clasificarlos es un punto de partida necesario desde el que iniciar un pensamiento reflexivo. De ese modo, se podrá orientar mejor su formación y se podrá capacitar a estos profesionales para hacer frente a esos dilemas.

Precisamente, el interés en profundizar en los dilemas éticos permitió, durante los años 2004 y 2005, realizar un proyecto de investigación titulado Valores morales y dilemas éticos en la intervención social. Este proyecto se llevó a cabo durante los años 2004 y 2005 con financiación del Gobierno de Navarra, y sus resultados fueron publicados en su día (Úriz y Ballestero, 2006; Úriz, Ballestero y Urien, 2007). En aquel momento, la principal preocupación era conocer qué tipo de dilemas éticos se encontraban las trabajadoras y los trabajadores sociales en el ejercicio diario de su profesión y centramos la investigación en los servicios sociales de base de Navarra. Los dilemas éticos con los que se encontraban más frecuentemente y que les causaban mayor preocupación eran los relativos a informes a terceros, a la propia realización de dichos informes (qué tipo de información incluir o no), al acceso a la historia social y, en definitiva, a todas las cuestiones relacionadas directamente con la confidencialidad y el tratamiento de la información.

Posteriormente a este estudio, se planteó ampliar geográficamente la investigación con el objetivo de averiguar cuáles son los principales dilemas éticos a los que se enfrentan los trabajadores y las trabajadoras sociales en toda España. De este modo, se ha realizado un estudio pionero sobre los dilemas éticos en trabajo social, en el que se sigue constatando que estos profesionales se encuentran con muchos y variados tipos de dilemas éticos a los que no siempre saben dar respuesta, bien por carecer de formación específica para ello, bien porque no disponen de las herramientas metodológicas necesarias. También se constata que muchos de los dilemas éticos que causaban mayor preocupación entre las trabajadoras y los trabajadores sociales de los servicios sociales de base de Navarra siguen siendo los que más frecuentemente han de resolver las trabajadoras y los trabajadores sociales de toda España.

\section{Metodología}

La metodología utilizada en el proyecto de investigación ha sido cuantitativa (basada en el envío de un cuestionario por correo postal) y también cualitativa (realización de diversas entrevistas semiestructuradas). A lo largo de este artículo, vamos a presentar parte de los resultados y su discusión a partir del análisis cuantitativo de los cuestionarios.

La población objeto de estudio es la compuesta por el colectivo de trabajadores sociales colegiados en España (Consejo General de Colegios Oficiales de Diplomados en Trabajo Social y Asistentes Sociales de España). La matriz de datos la compone una muestra de 700 trabajadores sociales. La unidad muestral 
es aleatoria y nominativa. Aplicando la fórmula del error muestral para poblaciones finitas, significa que, para un nivel de confianza de dos sigmas (95,5\%), donde $p=q=0,50$, donde el universo de referencia es de 8.505 profesionales y la muestra que se obtiene es $n=700$, el error muestral es de $\pm 3,5 \%$. El trabajo de campo se llevó a cabo durante el último trimestre de 2009.

El cuestionario utilizado está basado en el elaborado por la doctora Eileen Joan Ain (2003) en 2001 en Estados Unidos. Este cuestionario fue revisado y aprobado por los doctores Philip J. Boyle, director adjunto del Instituto Hastings (Vancouver, Canadá), vicepresidente y editor jefe del Centro Park Ridge (Chicago, Illinois); Jonathan D. Moreno, catedrático de Bioética en la Universidad de Virginia y director del Centro de Bioética, y Bárbara Ann Lieberman, experta en estadística y analista de programas del Ministerio de Sanidad y Servicios Sociales de EE. UU. Además, el estudio también fue sometido a la evaluación de un tribunal de expertos en ética del trabajo social: los doctores Norman Linzer y Charles Auerbach, catedráticos de Trabajo Social en la Universidad de Yeshiva (Nueva York) y Elaine Congress, catedrática de Servicios Sociales y directora de los programas de posgrado y doctorado de la Universidad de Fordham (Nueva York).

El cuestionario ha sido traducido, contextualizado y adaptado al ámbito español por los miembros del grupo EFIMEC de la Universidad Pública de Navarra. Este cuestionario había sido utilizado en el proyecto anterior de investigación realizado en Navarra, pero nuevamente se reformularon y se adaptaron algunas preguntas para tratar de contextualizarlo en el ámbito del trabajo social en España. Concretamente, se modificaron algunas preguntas que estaban basadas en el Código deontológico de la asociación norteamericana de trabajadores sociales (NASW, 1999) y se sustituyeron por otro tipo de preguntas vinculadas a cuestiones o artículos concretos presentes en el Código deontológico de trabajo social en España (1999).

El cuestionario está dividido en siete grandes bloques: datos personales, datos profesionales, código de ética, cuestiones éticas, dilemas éticos, dilemas éticos en su trabajo diario y formación en ética. Contiene algunas preguntas de escala Lickert y otras de carácter abierto. Para el presente artículo, se han utilizado las preguntas referidas a los dilemas éticos en su trabajo diario compuestas por una escala Likert de 18 variables. Para el proceso analítico, se ha empleado la versión número 18 del programa SPSS y se ha realizado un análisis factorial de correspondencias múltiples, donde interaccionan diversas variables asociadas en la investigación, así como un análisis de conglomerados (cluster analysis) para definir los grupos de interés en función de dichas variables.

\section{Resultados}

\section{Caracterización de la muestra}

La distribución por sexos de los profesionales del trabajo social en España está fuertemente feminizada: el $91 \%$ son mujeres y el $9 \%$, hombres, lo cual es un reflejo de lo que conocemos en las enseñanzas de trabajo social en España desde 
hace ya varios años. En cuanto a la distribución por edades, el $40 \%$ tiene entre 31 y 40 años y el $30 \%$ corresponde a la franja entre 41 y 50 años, mientras que el $17 \%$ tiene entre 20 y 30 años y el 13\% tiene más de 50 años. Vemos, pues, que el $70 \%$ de las profesionales se sitúa en la franja de edad de 31 a 50 años.

En cuanto a la experiencia profesional, el 38\% tiene entre 6 y 15 años de experiencia como trabajadora o trabajador social; el $28 \%$, entre 16 y 25 años de experiencia profesional; el 24\%, hasta 5 años de experiencia, y sólo un $10 \%$ tiene más de 25 años de experiencia. Respecto al lugar donde realizan su trabajo, la gran mayoría (un 70\%) corresponde a las administraciones públicas, mientras que el $14 \%$ lo realiza en alguna entidad privada y el $12 \%$, en alguna entidad concertada. De entre los restantes, el $1 \%$ está en paro, el $1 \%$ trabaja de forma autónoma y el $2 \%$ realiza otro tipo de actividad.

\section{Tipos de dilemas éticos}

Antes de presentar los tipos de dilemas éticos, hay que señalar que un $82 \%$ de los encuestados respondieron afirmativamente a la pregunta sobre si habían sido conscientes de algún dilema ético en su trabajo. Esto significa, en primer lugar, que las profesionales y los profesionales son conscientes de los dilemas éticos que les rodean y que, además, éstos son muy frecuentes en sus intervenciones laborales.

A continuación, se les preguntaba: «¿Ha experimentado en su trabajo algún dilema ético relacionado con alguno de los siguientes temas?». Se presentaban 18 tipos de dilemas éticos con cuatro posibles opciones de respuesta: «Nunca», «Pocas veces», «Algunas veces» $\mathrm{y}$ «Bastantes veces». Si agrupamos los que respondieron que habían tenido alguno de esos dilemas algunas o bastantes veces, obtenemos cinco tipos de dilemas que aparecen en más del $40 \%$ de los casos. De mayor a menor porcentaje, son los siguientes (gráfico 1): dilemas relativos al deber de informar a terceras personas, dilemas relacionados con el respeto a la autonomía del usuario, dilemas relacionados con la incompetencia de otra trabajadora social u otro profesional, dilemas relativos a la confidencialidad y dilemas relativos a la duración del tiempo de la intervención. Veamos de una forma más detallada en qué consisten estos dilemas.

\section{Dilema no 1: El deber de informar a terceras personas}

Entre los distintos tipos de dilemas planteados en el cuestionario, éste es el que alcanza el porcentaje más alto. El 48\% manifiesta haber tenido un dilema de esta clase algunas o bastantes veces. En este tipo de dilemas quedan comprometidos varios aspectos relevantes para la intervención profesional: el principio de la confidencialidad, el principio de beneficiencia y el tipo de relación que se establece entre trabajador social y usuario, en la que se define el tipo de relación comunicacional (dialógica) y el grado de libertad de la información. La información y su titularidad se convierten en un eje cotidiano de conflicto. La titularidad de la información pertenece siempre al usuario, pero hay circunstancias especiales en las que se justifica la ruptura de dicha confidencialidad, 
Gráfico 1. Tipos de dilemas éticos en trabajo social

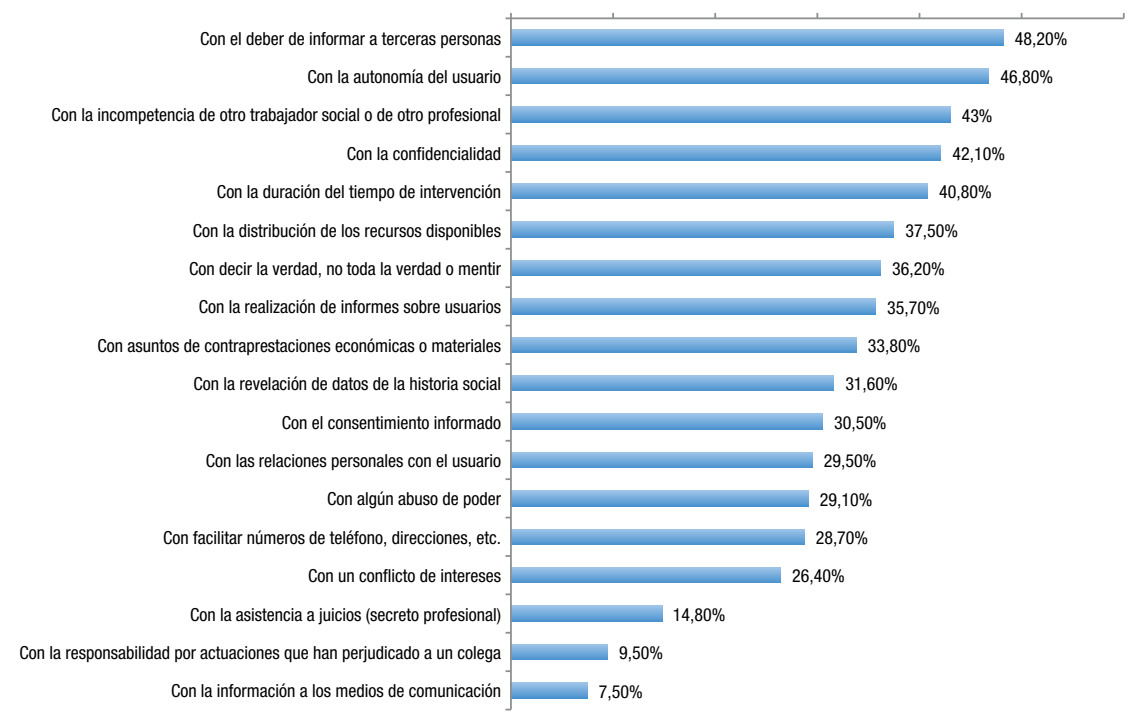

Fuente: elaboración propia.

como en los casos en los que la información confidencial pueda ser peligrosa para el usuario o para terceras personas.

En algunas ocasiones, personas que no están trabajando directamente en la intervención (responsables políticos de ayuntamientos u otros profesionales, etc.) solicitan algún tipo de información relativa a los usuarios. A veces, la demanda de información puede estar justificada, pero en otros casos estas demandas se hacen desde una supuesta posición de «poder» o superioridad de quien demanda la información y hace que las trabajadoras y los trabajadores sociales se sientan presionados para dar o no determinada información sobre programas, ayudas concedidas o sobre los usuarios. Los dilemas surgen cuando opinan que revelar esos datos puede suponer algún tipo de daño para los usuarios.

\section{Dilema no 2: La autonomía de los usuarios o usuarias}

El respeto a la libertad y a las decisiones de los usuarios y las usuarias es un aspecto fundamental de la práctica profesional del trabajo social. De hecho, el principio de autonomía de los usuarios y las usuarias suele ocupar un lugar muy importante en las diversas declaraciones y documentos del trabajo social. Sin embargo, un 46,5\% dice haber experimentado un dilema de esta clase algunas o bastantes veces, lo que indica que no siempre están muy claras las situaciones que limitan la autonomía o que, a veces, el respeto a la autonomía de los usuarios y las usuarias entra en conflicto con otro principio (el del bienestar, por ejemplo) y se crea un dilema ético. 
Un ejemplo muy frecuente de este tipo de situaciones dilemáticas es el de las profesionales que han tenido que denunciar un caso de violencia de género aún en contra de la voluntad de la mujer maltratada o en los casos de ingresos involuntarios en unidades psiquiátricas. En estos casos, es fundamental la evaluación del maltrato psicológico, así como de la capacidad o no de las personas para tomar sus propias decisiones.

\section{Dilema no 3: La incompetencia de otro trabajador o trabajadora social u otro profesional}

Este dato resulta un tanto sorprendente: un 43\% manifiesta haber tenido un dilema de esta clase algunas o bastantes veces. Se trata, en muchos casos, de situaciones de mala práctica laboral en las que el otro profesional no está cumpliendo las obligaciones derivadas de su competencia y la otra trabajadora o trabajador social duda sobre si ha de comunicárselo o no a un superior. Chomsky (1974) ya señalaba que la competencia profesional es un atributo que se articula y se perfecciona en su interacción con el entorno y en la que están muy presentes los valores profesionales, las habilidades laborales, las actitudes, las capacidades, los conocimientos, la representación de tareas y resultados, así como los métodos de intervención.

\section{Dilema no 4: La confidencialidad}

De un modo genérico, se puede afirmar que las trabajadoras y los trabajadores sociales saben que han de mantener la confidencialidad sobre la información relativa al usuario obtenida en el transcurso de la relación profesional, pero, pese a ello, el $41 \%$ dice haber experimentado un dilema ético en relación con la confidencialidad algunas o bastantes veces. Algunas dudas aparecen cuando la confidencialidad se concreta en situaciones más complejas, como el tipo de datos que se puede dar o no, las problemáticas en las que hay menores implicados, las situaciones en las que se teme perder la relación de confianza con las usuarias si se revela algún dato que ellas nos han pedido que no fuera revelado, etc.

\section{Dilema no 5: La duración del tiempo de la intervención}

Un 40\% manifiesta haber tenido un dilema de esta clase algunas o bastantes veces; por ejemplo: situaciones en las que han tenido que cerrar un expediente sin estar totalmente seguras de que eso fuera beneficioso para algunas de las personas implicadas en la intervención (menores, por ejemplo). También son especialmente significativos los problemas derivados del menor tiempo que los trabajadores y las trabajadoras sociales pueden dedicar a los usuarios. La mayor carga de trabajo que deben soportar los servicios de atención social debido a la situación de crisis, unida a la creciente burocratización a la que están siendo sometidos muchos de ellos, hacen que ese tiempo sea menor del que el professional consideraría necesario o deseable.

Hay otros cinco tipos de dilemas que aparecen entre un 30\% y un 37\% de los casos. Son los relativos a la distribución de recursos disponibles, dilemas sobre no decir toda la verdad o mentir, dilemas relacionados con contrapres- 
taciones económicas o materiales, dilemas relativos a la revelación de datos de la historia social y dilemas relacionados con el consentimiento informado. Analicemos brevemente cada uno de ellos.

\section{Dilema $n^{\circ}$ 6: La distribución de recursos disponibles}

Un $37 \%$ dice haber experimentado un dilema de esta clase algunas o bastantes veces. Cuando los recursos disponibles son escasos, es aún más frecuente encontrarse con dilemas relativos a la distribución de los mismos. Algunas trabajadoras sociales relatan dificultades en los criterios de aplicación de ayudas o en la distribución de recursos en salud mental, para personas discapacitadas, etc.

\section{Dilema no 7: Decir la verdad, no toda la verdad o mentir}

Un 36\% manifiesta haber tenido un dilema de esta clase algunas o bastantes veces. Algunas trabajadoras sociales refieren situaciones en las que, por ejemplo, descubrían que un usuario estaba mintiendo sobre algunos datos para conseguir una prestación determinada o no cumplía alguno de los requisitos establecidos para obtenerla. En estos casos, las trabajadoras sociales se debatían entre su obligación de comunicar todo tipo de situaciones fraudulentas o, por el contrario - y en casos de necesidad—, ocultar esa información para que el usuario obtuviera la prestación solicitada. A este respecto, conviene aclarar que las dudas de estas profesionales se daban en situaciones en las que consideraban que los usuarios o las usuarias necesitaban esas prestaciones, pese a no cumplir todos los requisitos establecidos.

\section{Dilema $n^{\circ}$ 8: La realización de informes sobre usuarios}

Una de las funciones más específicas de los trabajadores y las trabajadoras sociales españoles es la realización del informe social, donde tiene que quedar reflejada la situación en la que se encuentra el usuario (por ejemplo: ante la solicitud de una prestación determinada). El informe ha de incluir la valoración, el dictamen técnico y la propuesta de intervención por parte del trabajador social. Pues bien, aproximadamente un $35 \%$ de los profesionales ha tenido algún dilema ético en torno a la realización de informes sobre los usuarios, principalmente sobre su contenido y su redacción, así como sobre la titularidad del informe y el acceso al mismo, tanto por parte del usuario como de otros profesionales o instituciones.

\section{Dilema no 9: Los asuntos de contraprestaciones económicas o materiales}

Se refiere a situaciones en las que los usuarios u otras personas ofrecen algún tipo de contraprestación material o económica para intentar beneficiarse de algún servicio o prestación. El 34\% de las profesionales y los profesionales dicen haber experimentado un dilema de esta clase algunas o bastantes veces.

\section{Dilema no 10: La revelación de datos de la historia social}

Una de las funciones de las trabajadoras y de los trabajadores sociales es elaborar la historia social, que refleja el objeto, la valoración, el dictamen técnico 
y la propuesta de intervención profesional. Un 31\% manifiesta haber tenido algunas o bastantes veces un dilema ético referido a si deberían o no revelar algún dato de la historia social a los usuarios o usuarias o a otras personas. A este respecto, hay que tener en cuenta que los usuarios y las usuarias pueden solicitar el acceso a la historia social, por lo que hay que tener especial cuidado en que aparezcan datos técnicos y valoraciones objetivas, además de evitar opiniones subjetivas o valoraciones personales.

En cuanto a las informaciones que se aportan a otros profesionales, éstas deben limitarse solo a los aspectos estrictamente necesarios para la intervención relativa al tema concreto de que se trate, respetando siempre el secreto profesional, tal como aparece reflejado en el artículo 48 del Código deontológico de trabajo social en España.

\section{Dilema no 11: El consentimiento informado}

Una de las obligaciones de las trabajadoras y los trabajadores sociales es obtener el consentimiento del usuario o la usuaria para muchos aspectos de la intervención: iniciar un tratamiento determinado, utilizar la información sobre su persona intentando conseguir una mayor coordinación con otros profesionales o servicios, etc. El consentimiento se deriva directamente del principio de autonomía, pues si la persona es autónoma, es capaz de dar su consentimiento para, por ejemplo, iniciar un tratamiento determinado.

Pero el consentimiento ha de ser real y efectivo y, sobre todo, informado. Esto significa que se ha de explicar realmente a los usuarios y a las usuarias los distintos programas, las ventajas y desventajas de los mismos, etc., asegurándose de que los usuarios realmente han comprendido la información que se les está transmitiendo. La gran mayoría de las trabajadoras y los trabajadores sociales son conscientes de la importancia de obtener el consentimiento de los usuarios, pero, pese a ello, un 30\% manifiesta haber tenido un dilema ético relacionado con la obtención del consentimiento informado algunas o bastantes veces.

\section{Otros dilemas éticos menos frecuentes}

Finalmente, los trabajadores y las trabajadoras sociales manifiestan haber tenido menos frecuentemente dilemas en consonancia con las relaciones personales con los usuarios, los abusos de poder, la entrega de números de teléfono o direcciones, los conflictos de intereses, la asistencia a juicios, la responsabilidad por actuaciones que han perjudicado a un colega y la información a los medios de comunicación.

\section{Análisis factorial: una nueva clasificación de los tipos de dilemas éticos}

A partir de los resultados obtenidos en torno a los distintos dilemas éticos, se ha realizado un análisis factorial que ha reunido a los diferentes temas éticos que aparecen en el desempeño profesional en torno a cuatro grandes grupos, de tal forma que podemos aportar una nueva clasificación de los mismos. 
Tabla 2. Análisis factorial de los grupos de dilemas éticos

\begin{tabular}{|c|c|c|c|c|c|}
\hline & \multicolumn{4}{|c|}{ Matriz de componentes rotados (a) } \\
\hline & & \multicolumn{4}{|c|}{ Factores } \\
\hline & & 1 & 2 & 3 & 4 \\
\hline \multirow[t]{7}{*}{$\begin{array}{l}\text { FACTOR } 1 . \\
\text { Información }\end{array}$} & $\begin{array}{l}\text { Revelación de datos de la historia } \\
\text { social }\end{array}$ & 0,769 & 0,092 & 0,224 & $-0,023$ \\
\hline & $\begin{array}{l}\text { Deber de informar a terceras } \\
\text { personas }\end{array}$ & 0,737 & 0,107 & 0,003 & 0,002 \\
\hline & Confidencialidad & 0,700 & 0,296 & 0,010 & 0,098 \\
\hline & $\begin{array}{l}\text { Facilitación de números de teléfono, } \\
\text { de direcciones personales }\end{array}$ & 0,627 & 0,026 & 0,175 & 0,310 \\
\hline & $\begin{array}{l}\text { Decir la verdad, no toda la verdad } \\
\text { o mentir }\end{array}$ & 0,503 & 0,310 & 0,212 & 0,096 \\
\hline & Consentimiento informado & 0,489 & 0,214 & 0,080 & 0,439 \\
\hline & $\begin{array}{l}\text { Relacionado con la realización } \\
\text { de informes sobre usuarios }\end{array}$ & 0,479 & 0,453 & 0,177 & $-0,108$ \\
\hline \multirow{6}{*}{$\begin{array}{l}\text { FACTOR } 2 . \\
\text { Aspectos intrínsecos } \\
\text { a la intervención }\end{array}$} & $\begin{array}{l}\text { Duración del tiempo } \\
\text { de la intervención }\end{array}$ & 0,078 & 0,708 & 0,121 & 0,035 \\
\hline & $\begin{array}{l}\text { Distribución de los recursos } \\
\text { disponibles }\end{array}$ & 0,068 & 0,645 & 0,302 & $-0,035$ \\
\hline & $\begin{array}{l}\text { Asuntos de contraprestaciones } \\
\text { económicas o materiales }\end{array}$ & 0,198 & 0,621 & $-0,010$ & 0,250 \\
\hline & Conflicto de intereses & 0,191 & 0,584 & 0,254 & 0,001 \\
\hline & Abuso de poder & 0,191 & 0,570 & 0,041 & 0,073 \\
\hline & Autonomía del usuario & 0,463 & 0,506 & $-0,051$ & 0,124 \\
\hline \multirow{4}{*}{$\begin{array}{l}\text { FACTOR } 3 . \\
\text { Aspectos extrínsecos } \\
\text { a la intervención }\end{array}$} & Responsabilidad por actuaciones & 0,196 & 0,040 & 0,722 & 0,273 \\
\hline & $\begin{array}{l}\text { que han perjudicado a un cole } \\
\text { Información a los medios } \\
\text { de comunicación }\end{array}$ & 0,133 & 0,129 & 0,679 & $-0,080$ \\
\hline & $\begin{array}{l}\text { Incompetencia de otro trabajador } \\
\text { social o de otro profesional }\end{array}$ & $-0,065$ & 0,373 & 0,524 & 0,205 \\
\hline & $\begin{array}{l}\text { Asistencia a juicios (secreto } \\
\text { profesional) }\end{array}$ & 0,333 & 0,273 & 0,448 & $-0,425$ \\
\hline $\begin{array}{l}\text { FACTOR } 4 . \\
\text { Relación personal } \\
\text { con el usuario }\end{array}$ & Relaciones personales con el usuario & 0,180 & 0,171 & 0,161 & 0,735 \\
\hline
\end{tabular}

Fuente: elaboración propia.

Recordemos que el análisis factorial que hemos realizado se basa en los datos facilitados por los profesionales y de dicho análisis surgen cuatro categorías:

1. Factor 1. Dilemas éticos relacionados con la información. Agrupa todos aquellos dilemas éticos que se encuentran relacionados con aspectos que tienen que ver con el manejo de datos relevantes utilizados en torno a un caso social: la revelación de datos de la historia social, el deber de informar 
a terceras personas, la confidencialidad, la facilitación de datos personales, el hecho de decir la verdad, el consentimiento informado y la realización de informes sobre usuarios.

2. Factor 2. Relacionados con aspectos intrínsecos a la intervención profesional. Temas propios de la ejecución de la intervención social y que atañen a aspectos como la duración del tiempo de intervención, la distribución de los recursos disponibles, las contraprestaciones económicas o materiales, los conflictos de intereses, el abuso de poder o la autonomía del usuario.

3. Factor 3. Relacionados con aspectos extrínsecos a la intervención profesional. Cuestiones que no pertenecen propiamente a la intervención profesional, pero que se derivan de ella. Además, el uso del prefijo extra-conlleva que se encuentren fuera, que sean colaterales a la intervención propiamente dicha. Temas relacionados con la responsabilidad por actuaciones que han perjudicado a un colega, por la información a los medios de comunicación, por la incompetencia de otros profesionales o por la asistencia a juicios.

4. Factor 4. Relacionados con aspectos relacionales (conexión entre trabajador social y usuario). Se refiere a los dilemas éticos que se producen como producto del tipo de relación que se establece entre trabajador social y usuario.

\section{Análisis de conglomerados (cluster analysis)}

Una vez llevada a cabo esta reducción dimensional, se procedió a generar una clasificación tipológica de los profesionales según su posición factorial. Con ello se pretende observar si existen diferentes tipos de profesionales a la hora de enfrentarse a unos u otros dilemas éticos. Para ello, hemos procedido a la realización de un estudio de conglomerados no jerárquico ( $k$ medias). Para la realización de un análisis de conglomerados, se requiere tener en cuenta tres aspectos que son fundamentales a la hora de elegir las variables que se utilizan, tal y como señala Martínez Ramos (1984): que las variables estén intercorrelacionadas, que la unidad de medida sea la misma para todas las variables analizadas y que el número de variables no sea demasiado grande. Los datos del presente análisis cumplen todos estos requisitos. Hemos llevado a cabo un análisis de varianza con el fin de conocer hasta qué punto cada uno de los factores permite una diferenciación significativa de los individuos de cada cluster.

El resultado (tabla 3) demuestra que los factores elegidos producen diferencias signficativas en la concreción de los cinco clusters.

Tabla 3. Diferencias significativas de los factores en cada cluster (análisis de varianza)

\begin{tabular}{lcc}
\hline Factor & F & Sig. \\
\hline 1. Temas dilemas éticos: información & 8,373 & 0,000 \\
2. Temas dilemas éticos: extrínsecos a la intervención & 5,598 & 0,000 \\
3. Temas dilemas éticos: intrínsecos a la intervención & 7,997 & 0,000 \\
4. Temas dilemas éticos: relaciones personales & 5,208 & 0,000 \\
\hline
\end{tabular}

Fuente: elaboración propia. 
Como técnica de agrupamiento, se ha elegido la clasificación no jerárquica ( $\mathrm{k}$-means) y los resultados obtenidos han sido validados mediante análisis discriminante (bondad de la clasificación obtenida). Dicho análisis señala que clasifica correctamente el 96,1\% de los casos. De la realización de dicho estudio, emergen cinco grupos característicos:

Tipo 1. Externos: representa el 26,5\% de los profesionales y tienen dilemas éticos extrínsecos a la intervención. Se trata de profesionales que se muestran seguros en el manejo de los dilemas éticos más propios de la intervención profesional (intrínsecos, informacionales) y que, sin embargo, son los aspectos externos los que mayor distorsión les generan. Sobre todo, en este apartado, destacan con especial relevancia los dilemas éticos que tienen que ver con la incompetencia de otro profesional o de otro trabajador social. Este es un aspecto preocupante y que pone el acento en la necesidad de mejorar las capacidades del trabajo multidisciplinario, del trabajo en equipo y, sobre todo, de la coordinación de los profesionales en la gestión de casos. Este colectivo tiene una marcada correlación con algunas variables, como son la problemática que atienden, el lugar del trabajo en el que desempeñan su acción y, en menor medida, la experiencia profesional. Es especialmente significativa la presencia de este tipo de dilemas éticos entre los profesionales que trabajan en el ámbito de la violencia y de la marginación social (violencia, violencia contra las mujeres, prostitución, racismo e inmigración) $(42,9 \%)$ y entre aquellos que trabajan

Gráfico 2. Cluster 1. Externos (26,5\%)

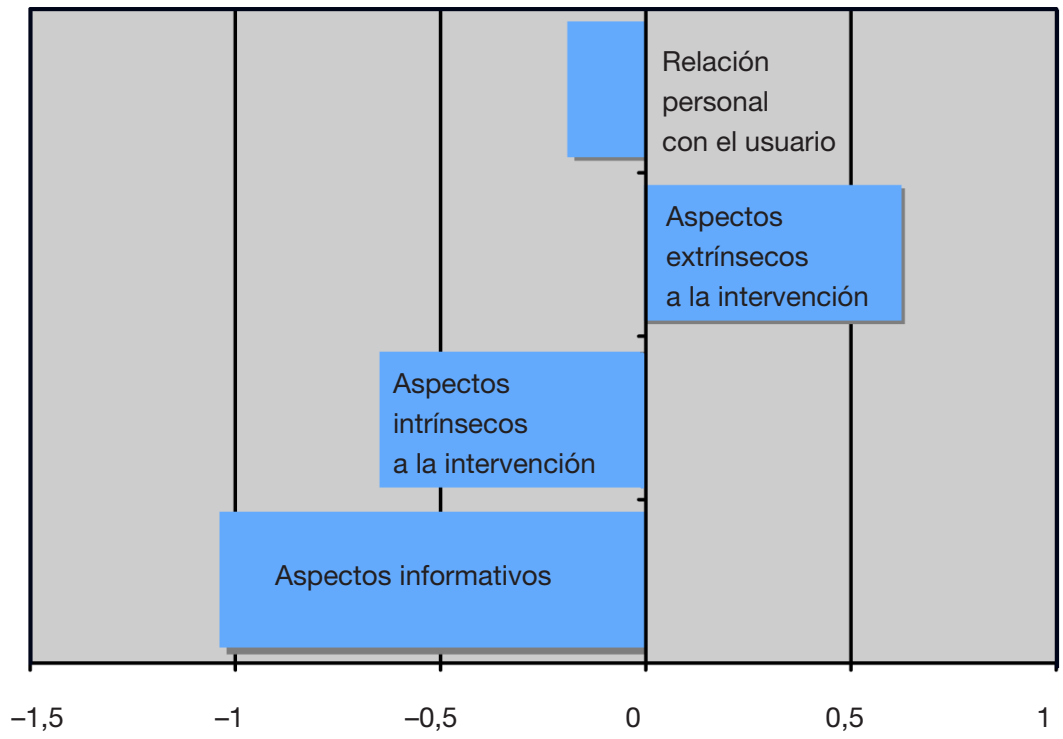

Fuente: elaboración propia. 
con las necesidades individuales (desempleo, vivienda, alojamiento, problemas económicos, renta básica) (31,1\%). Lo delicado de ciertas intervenciones sobre todo en el ámbito de la violencia y de la marginación social—, derivado de la complejidad de factores y de profesionales que intervienen en las mismas, justifica una mayor presencia de este tipo de dilemas éticos relacionados con la asistencia a juicios, la información a medios de comunicación y la dificultad con otros colegas (incompetencia profesional propia o de otros).

Igualmente, se encuentra una mayor presencia de este tipo de dilemas éticos entre los profesionales que desempeñan su labor en el ámbito concertado $(40,7 \%)$ y en el autónomo $(42,8 \%)$.

Tipo 2. Compañeros: numéricamente, es el segundo tipo en importancia y agrupa al $24,4 \%$ de los profesionales. Son trabajadores sociales que tienen dilemas éticos propios de mantener un tipo de relación con el usuario que va más allá de los límites que establece una conexión meramente profesional. La relación de ayuda es un proceso consustancial al trabajo social en cualquiera de sus niveles: individual, familiar, grupal o comunitario. La relación entre trabajador social y persona que necesita la ayuda (cliente, usuario, ciudadano, paciente) es interactiva y, para que sea efectiva (propiciar cambios), es necesario que sea continuada y bidireccional. Perlman (1980: 13) establece que «una buena relación de ayuda es la que provee estímulos y crecimiento, respeta y alimenta la individualidad del otro». Esa bidireccionalidad establece una vinculación profesional que es una relación especial dentro del contexto de la intervención. En ocasiones, esa vinculación - producto de los factores humanos que intervienen en ella- se convierte en una vinculación humana más que en una vinculación estrictamente profesional y ahí es donde aparecen los dilemas éticos. No es de extrañar que, en este tipo de profesionales, también aparezca como elemento de dificultad, aunque en menor medida, el tratamiento de la información del usuario. La excesiva confianza o la excesiva vinculación contribuyen, además, a que se difuminen algunos límites en el manejo de ciertas informaciones relativas al caso de intervención, lo que hace que igualmente presenten valores positivos en el análisis estadístico.

Este colectivo tiene una marcada correlación con variables como las siguientes:

- Problemática que atienden. Se observa un mayor peso de este tipo de dilemas en los profesionales que trabajan problemáticas relacionadas con la salud mental y las denominadas «enfermedades sociales» (adicciones, ludopatías) (35,1\%). No es de extrañar que se dé en los profesionales que trabajan en el ámbito de la exclusión social y de las enfermedades sociales, puesto que desempeñan su labor de manera muy próxima al usuario, y la acogida, la escucha inicial, la receptividad, el diálogo, la confianza, la sinceridad y la autenticidad son piezas maestras para la reconstrucción y el reencuentro personal. Es determinante un mayor grado de cercanía con el usuario y es desde ahí desde donde se explica que sean estos profesionales los que mencionen tener más dilemas en este sentido. 
Gráfico 3. Cluster 2. Compañeros (24,3\%)

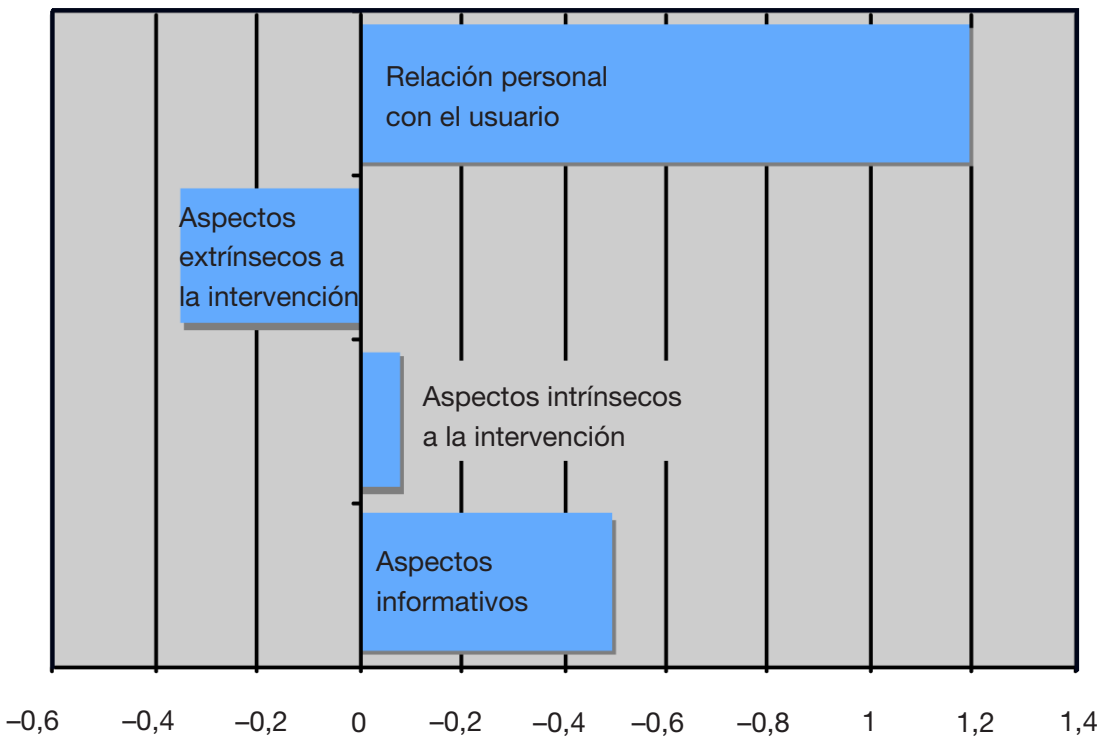

Fuente: elaboración propia.

- Lugar de trabajo. Se observa que es en las entidades concertadas donde se da un mayor grado de presencia de este tipo de dilemas éticos (36,3\%), el cual está claramente correlacionado con el tipo de problemática con la que este tipo de entidades intervienen.

- Sexo. Son los hombres $(33,9 \%)$ los que significativamente presentan una mayor dificultad ética en este apartado.

Tipo 3. Esenciales: este cluster agrupa al 17,8\% de los profesionales encuestados. Se denominan así por ser los que encuentran dilemas éticos referidos a la "esencia» de la intervención, a aquellos dilemas propios derivados directamente de la intervención profesional directa y de su ejecución. Alude a los dilemas éticos que se producen en la acción profesional distintiva del trabajo social, que está orientada a la ayuda a las personas y sus entornos para que venzan los obstáculos que impiden su crecimiento, su desarrollo y su funcionamiento adaptativo. Es muy clara su ubicación, ya que los datos estadísticos informan de que sólo tienen dilemas éticos de esta índole, y no presentan dificultades éticas en el ámbito relacional, ni con los aspectos éticos externos a la intervención.

Este colectivo tiene una marcada correlación con variables como:

- La problemática que atienden. Son profesionales que trabajan con problemáticas de intervención que atañen a las necesidades individuales (aloja- 
Gráfico 4. Cluster 3. Esenciales (17,4\%)

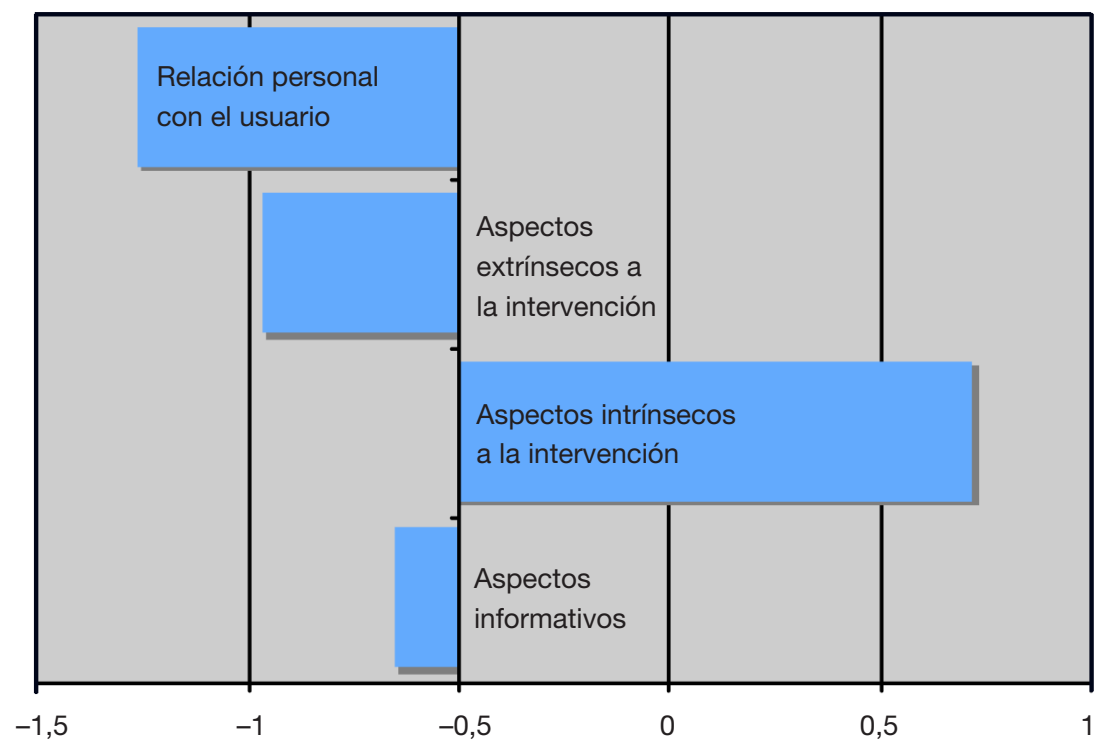

Fuente: elaboración propia.

miento, vivienda, desempleo, renta básica) y aquellos que atienden a los procesos interpersonales problemáticos, los que significativamente manifiestan mayor peso de estos dilemas éticos en su práctica profesional. Esto tiene que ver claramente con las características de la intervención que realizan, centrada en mejorar la interacción, la comunicación de las personas con los sistemas que les rodean, mejorar las capacidades de la gente que atienden para solucionar sus problemas y enlazar a las personas con los sistemas de recursos y servicios.

- Lugar de trabajo. Es en los trabajadores de la Administración pública donde se produce un mayor peso de este tipo de dilemas éticos.

Tipo 4. Complejos: clasifica al 11,8\% de los profesionales. Se denominan así por ser los que, en su práctica cotidiana, se enfrentan a dilemas éticos de diversa procedencia, lo que configura el desempeño de una labor ética compleja. La complejidad viene definida principalmente por tener que abordar, con mayor o menor frecuencia, dilemas éticos de todas las tipologías, a excepción de los dilemas éticos que tienen que ver con el factor relacional como denominador común. Analizando las puntuaciones factoriales, destacan sobre todo los dilemas éticos extrínsecos (externos a la intervención profesional), seguidos de los dilemas informativos y, finalmente, los dilemas intrínsecos.

Por otro lado, este colectivo se encuentra sobrerrepresentado por profesionales que trabajan con el conjunto de problemáticas sociales y que, en la 
Gráfico 5. Cluster 4. Complejos (11,8\%)

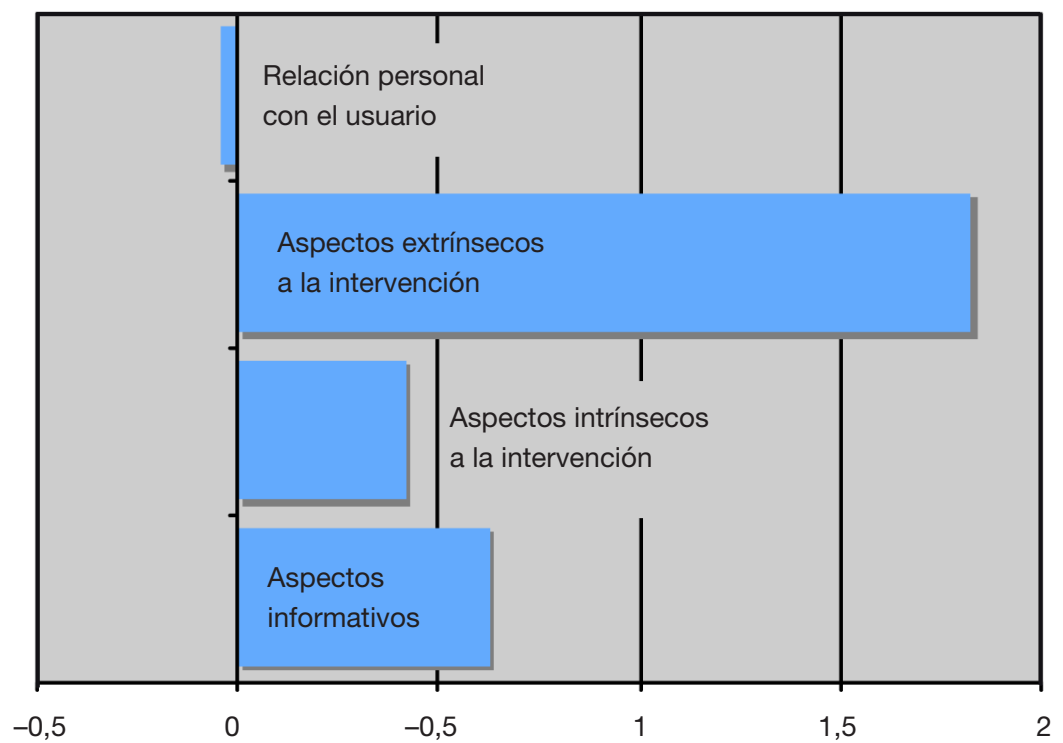

Fuente: elaboración propia.

disciplina del trabajo social, son denominados «trabajadores sociales generalistas o polivalentes». Son profesionales que llevan a cabo una actuación múltiple y que engloba muchas facetas de la intervención social: proporcionan diversos servicios básicos para ayudar a individuos, familias y comunidades a comprender y resolver sus problemas, actúan como catalizadores de los problemas sociales en las diferentes áreas de población avanzando la promoción y la utilización de los servicios sociales a través de la coordinación de los recursos y las necesidades de la comunidad y estimulan la participación de individuos, grupos y comunidades en la institucionalización del bienestar social, promoviendo la creación de organizaciones comunitarias. Esta labor compleja y múltiple es razonable que conlleve una complejidad ética claramente revelada en los resultados obtenidos.

Igualmente, encontramos sobrerrepresentación de este conjunto en la Administración pública, algo lógico, porque es donde generalmente se desempeña este tipo de intervención.

Tipo 5. Reveladores: agrupa el 19,9\% de los profesionales encuestados. Este cluster se denomina así por tratarse de profesionales que se encuentran con dilemas éticos relacionados exclusivamente con el tratamiento de la información y con la revelación de datos. El resto de tipos de dilemas éticos apenas tienen relevancia y registran valores estadísticos muy negativos.

$\mathrm{Al}$ igual que en los conjuntos anteriores, éste tiene una marcada correlación con algunas variables: 
Tabla 4. Rasgos de los tipos de profesionales según los dilemas éticos a los que se enfrentan

\begin{tabular}{llcccccc}
\hline & & Externos & Compañeros & Esenciales & Complejos & Reveladores & Total \\
\hline$N=700$ & Media & 26,6 & 24,4 & 17,4 & 11,8 & 19,9 & 100 \\
\hline Sexo & Mujer & 26,7 & 23,5 & 17,3 & 11,8 & 20,7 & 100 \\
& Hombre & 25,0 & 33,9 & 17,9 & 12,5 & 10,7 & 100 \\
\hline Experiencia & Hasta 5 años & 30,4 & 28,0 & 13,7 & 7,1 & 20,8 & 100 \\
profesional & Entre 6 y 15 años & 24,7 & 25,1 & 19,8 & 13,1 & 17,3 & 100 \\
& Entre 16 y 25 años & 26,4 & 20,2 & 17,4 & 13,5 & 22,5 & 100 \\
& Más de 25 años & 23,0 & 23,6 & 18,2 & 14,6 & 20,6 & 100 \\
Problemática & Violencia y marginación & 42,9 & 26,4 & 6,6 & 8,7 & 15,4 & 100 \\
que atienden & Enfermedades sociales & 19,8 & 35,1 & 14,1 & 5,6 & 25,4 & 100 \\
& Necesidades individuales & 31,1 & 21,2 & 23,7 & 4,3 & 19,7 & 100 \\
& Procesos interpersonales & 17,9 & 22,1 & 22,1 & 8,5 & 29,4 & 100 \\
& problemáticos & & & & & & \\
& Generalistas & 18,5 & 23,5 & 18,5 & 24,1 & 15,4 & 100 \\
\hline \multirow{2}{*}{ Lugar } & Administración pública & 23,8 & 22,2 & 22,2 & 12,1 & 19,7 & 100 \\
de trabajo & Entidad concertada & 40,7 & 19,8 & 9,9 & 9,8 & 19,8 & 100 \\
& Entidad privada & 25,3 & 36,3 & 11,0 & 5,4 & 22,0 & 100 \\
& Autónomo & 42,8 & 28,6 & 14,3 & 0 & 14,3 & 100 \\
\hline
\end{tabular}

Fuente: elaboración propia.

- Problemática con la que trabajan. Se observa una mayor sobrerrepresentación de profesionales que trabajan con problemáticas relacionadas con la intervención en procesos interpersonales problemáticos (conflictos familiares y problemas de conducta) y aquellos que trabajan con problemáticas relacionadas con la salud mental y las enfermedades sociales (ludopatías y adicciones).

- Igualmente, se observa una mayor sobrerrepresentación en el caso de las mujeres respecto de los hombres: un 20,7\% de las mujeres frente a un $10,7 \%$ de los varones.

- Entidades privadas. Es en este tipo de entidades en las que se observa una ligera sobrerrepresentación.

\section{Discusión}

En primer lugar, hemos confirmado nuestra hipótesis inicial de que los trabajadores y las trabajadoras sociales han de enfrentarse, en su trabajo diario, a una gran cantidad y variedad de dilemas éticos. También nos planteábamos, como otra hipótesis, que muchos de esos dilemas éticos estarían relacionados con los principios éticos de autonomía y confidencialidad de la información, lo cual ha sido corroborado mediante los resultados obtenidos. En cambio, no pensábamos que los dilemas éticos en relación con la incompetencia de otros profesionales fueran a tener tanta relevancia. 
Tabla 5. Clasificación, según su importancia, de los dilemas éticos que aparecen en el desempeño profesional. Comparación entre España y Estados Unidos

\begin{tabular}{|c|c|c|}
\hline $\begin{array}{l}\text { Clasificación de los dilemas éticos. } \\
\text { España (Grupo Efimec) (1) }\end{array}$ & $\begin{array}{l}\text { Algunas o } \\
\text { bastantes } \\
\text { veces }\end{array}$ & $\begin{array}{c}\text { Clasificación «Top Ten Ethic Dilemmas». } \\
\text { EE. UU. (NASW) (2) }\end{array}$ \\
\hline $\begin{array}{l}1 \text { Con el deber de informar a terceras } \\
\text { personas }\end{array}$ & $48,2 \%$ & 1. Confidentiality. \\
\hline 2 Con la autonomía del usuario & $46,8 \%$ & $\begin{array}{l}\text { 2. Sexual relationships with clients and } \\
\text { with prior clients. }\end{array}$ \\
\hline $\begin{array}{l}3 \text { Con la incompetencia de otro } \\
\text { trabajador social o de otro profesional }\end{array}$ & $43,0 \%$ & $\begin{array}{l}\text { 3. Inappropriate physical contact with } \\
\text { clients. }\end{array}$ \\
\hline 4 Con la confidencialidad & $42,1 \%$ & 4. Autonomy issues. \\
\hline $\begin{array}{l}5 \text { Con la duración del tiempo de la } \\
\text { intervención }\end{array}$ & $40,8 \%$ & 5. Conflicts of interest. \\
\hline $\begin{array}{l}6 \text { Con la distribución de los recursos } \\
\text { disponibles }\end{array}$ & $37,5 \%$ & $\begin{array}{l}\text { 6. Scarcity of resources at worksites } \\
\text { and other worksite issues which } \\
\text { interfere with serving clients. }\end{array}$ \\
\hline $\begin{array}{l}7 \text { Con decir la verdad, no toda la ver- } \\
\text { dad o mentir }\end{array}$ & $36,2 \%$ & 7. Impaired colleague issues. \\
\hline $\begin{array}{l}8 \text { Con la realización de informes sobre } \\
\text { usuarios }\end{array}$ & $35,7 \%$ & 8. Unethical conduct of colleagues. \\
\hline $\begin{array}{l}9 \text { Con asuntos de contraprestaciones } \\
\text { económicas o materiales }\end{array}$ & $33,8 \%$ & $\begin{array}{l}\text { 9. Practice competence, misrepresenta- } \\
\text { tion of expertise, and dishonesty. }\end{array}$ \\
\hline $\begin{array}{l}10 \text { Con la revelación de datos de la his- } \\
\text { toria social }\end{array}$ & $31,6 \%$ & $\begin{array}{l}\text { 10. Cultural competence and social } \\
\text { diversity and issues of discrimination. }\end{array}$ \\
\hline 11 Con el consentimiento informado & $30,5 \%$ & \\
\hline $\begin{array}{l}12 \text { Con las relaciones personales con el } \\
\text { usuario }\end{array}$ & $29,5 \%$ & \\
\hline 13 Con algún abuso de poder & $29,1 \%$ & \\
\hline $\begin{array}{l}14 \text { Con facilitar números de teléfono, } \\
\text { direcciones, etc. }\end{array}$ & $28,7 \%$ & \\
\hline 15 Con un conflicto de intereses & $26,4 \%$ & \\
\hline $\begin{array}{l}16 \text { Con la asistencia a juicios (secreto } \\
\text { profesional) }\end{array}$ & $14,8 \%$ & \\
\hline $\begin{array}{l}17 \text { Con la responsabilidad por actuacio- } \\
\text { nes que han perjudicado a un colega }\end{array}$ & $9,5 \%$ & \\
\hline $\begin{array}{l}18 \text { Con la información a los medios de } \\
\text { comunicación }\end{array}$ & $7,5 \%$ & \\
\hline
\end{tabular}

Fuente 1: elaboración propia.

Fuente 2: NASW (1998).

Los datos sobre dilemas éticos del trabajo social en España contrastan en algunos temas con los de la NASW (National Association of Social Workers) de 1998 respecto de los diez dilemas éticos más frecuentes a los que se enfrentan los trabajadores sociales en Estados Unidos. Es necesario señalar que las similitudes entre ambas clasificaciones se refieren a dos aspectos clave, transversales e identificables en toda intervención profesional del trabajo social, como 
son la autonomía del usuario y la confidencialidad. Las principales diferencias estriban en un mayor peso en EE. UU. de los dilemas éticos relacionados con la relación con el usuario (relaciones sexuales o contacto físico inapropiado con los clientes), de modo que se convierte en el segundo dilema ético en importancia, después de la confidencialidad. También tienen mayor peso que en España los dilemas éticos relacionados con los conflictos de intereses (relaciones duales y múltiples). Por el contrario, en nuestro país, destacan por tener una posición más elevada en la clasificación los dilemas relacionados con la incompetencia profesional. Ello apunta a que los factores «estructurales» y "contextuales» que configuran el ejercicio de la profesión en cada uno de los países parecen tener influencia en el tipo de dilemas éticos a los que habitualmente deben enfrentarse los trabajadores y las trabajadoras sociales.

También observamos que siguen siendo muy frecuentes algunos de los dilemas éticos identificados en el estudio realizado en los servicios sociales de base de Navarra, como los relativos a informar o no a terceras personas y los relacionados con la confidencialidad (Úriz, Ballestero y Urien, 2007). En cambio, ahora resultan menos importantes los dilemas relativos a la realización del informe social (probablemente porque es un tema en el que se viene incidiendo mucho en la formación de los trabajadores sociales) y, en cambio, son más importantes los dilemas relativos a la incompetencia profesional.

Linzer, Conboy y Ain (2003) realizaron en Israel un estudio similar al que presentamos en este artículo. Tenían como uno de sus principales objetivos identificar los tipos de dilemas éticos que tienen que resolver los trabajadores sociales israelíes. Porcentualmente, y en orden de mayor a menor frecuencia, los seis primeros tipos de dilemas son: los relativos a la confidencialidad (más del $70 \%$ ), al deber de advertir a una persona de un posible daño grave (casi un $60 \%$ de los casos), la preocupación en torno a la peor calidad del servicio que se presta a los usuarios (debido al número de éstos que los profesionales han de atender) (55\%), dilemas en torno a advertir o no sobre la incompetencia de otros profesionales (55\%), dilemas sobre la forma de obtener el consentimiento informado (45\%) y dilemas en torno a decir o no toda la verdad o a mentir (también aproximadamente un 45\%).

Si comparamos el estudio de Linzer, Conboy y Ain en Israel con el que hemos realizado nosotros en España, podemos decir que los dilemas éticos en torno a la confidencialidad (y las situaciones en las que ésta se puede romper) son importantes en ambos países, aunque todavía lo son más en Israel que en España. Además, los dilemas sobre la incompetencia de otros profesionales ocupan el tercer lugar en ambos países. En cuanto a los dilemas en torno al consentimiento informado y a decir o no toda la verdad, son más frecuentes en Israel que en España (en nuestro país ocupan un lugar intermedio). Por el contrario, los dilemas en torno al respeto a la autonomía de los usuarios son más importantes en España (46,8\%) que en Israel (no llegan al 20\%).

En cuanto a las posibles formas de clasificar dilemas éticos, no hay una única clasificación general, sino que los diversos especialistas en el tema los agrupan siguiendo diversos criterios. Strom-Gottfried (2006), por ejemplo, 
distingue entre dilemas según se realice un trabajo con individuos, familias o con grupos. Por su parte, Reamer (1990) realiza una clasificación de los principales dilemas éticos en torno a tres grandes categorías:

1. Los dilemas éticos relacionados con la intervención con individuos, grupos y familias, donde los principales temas éticos de dificultad son los referidos a la confidencialidad, la información, la autodeterminación del usuario, el paternalismo y el consentimiento informado.

2. Los dilemas derivados del diseño y la administración de programas y políticas de bienestar, donde los principales dilemas éticos son los relativos a la distribución de recursos limitados, el derecho de los individuos al bienestar y la cobertura de personas en situación de extrema necesidad.

3. Los derivados de las relaciones con los profesionales o colegas, donde los principales dilemas éticos se refieren a la incompetencia profesional, el uso de la información relativa a los usuarios, los conflictos con las normativas de cada institución, etc.

Una de las aportaciones principales de este artículo reside en la clasificación de los dilemas éticos agrupándolos en cuatro factores: dilemas relacionados con la información, dilemas intrínsecos a la intervención, dilemas extrínsecos a la intervención profesional y dilemas referidos a la relación entre trabajador social y usuario. Además, se ha identificado en qué tipo de profesionales se dan con mayor frecuencia cada uno de esos grupos de dilemas. Destaca también que es la primera vez que se realiza un estudio de estas características de ámbito nacional.

\section{Conclusiones}

Una primera conclusión indica que las trabajadoras y los trabajadores sociales españoles reconocen -en un porcentaje muy alto (un 82\%) - haber experimentado algún dilema ético en su ejercicio profesional.

La situación que provoca más dilemas éticos es el deber o no de informar a terceras personas. A este respecto, las trabajadoras y los trabajadores sociales deberían tener muy en cuenta que «la documentación de trabajo está sujeta a criterios de confidencialidad, por lo que su uso queda limitado por y para el objetivo profesional de que se trate» (Código deontológico de trabajo social en España, artículo 29). Cuando otras trabajadoras sociales, otros profesionales de otras instituciones e incluso algunos responsables políticos demanden cierta información, habrá que evaluar qué tipo de información se está demandando, así como si los profesionales u otras personas que la solicitan están o no implicadas en la intervención con los usuarios. Para ello contamos con una herramienta legal muy útil: la Ley Orgánica 15/1999, de 13 de diciembre, de protección de datos de carácter personal.

También en el artículo 48 del Código deontológico de trabajo social en España se afirma lo siguiente: «El diplomado en trabajo social/asistente social debe 
limitar las informaciones que aporta a sus colegas y a otros profesionales tan sólo a los elementos que considere estrictamente indispensables para la consecución del objetivo común, respetando el secreto profesional».

El segundo de los dilemas - en orden de mayor a menor importancia- es el relativo al respeto a la autonomía de las usuarias y los usuarios. El principio de autonomía suele ser considerado uno de los principios éticos más importantes en trabajo social. El artículo 5 del Código deontológico de trabajo social dice que «cada individuo tiene derecho a la autorrealización». Sin embargo, al igual que otros principios éticos, la autonomía, la autorrealización o la autodeterminación de los usuarios y las usuarias no es un principio ético absoluto, sino que tiene limitaciones. Una de las principales es la que señala el propio artículo 5 del Código deontológico, que limita el derecho a la autorrealización de los usuarios «hasta donde no interfiera en el derecho de los demás». El riesgo grave para la vida, la salud o la integridad de terceras personas limita el principio de autonomía, así como ese mismo riesgo grave para el propio usuario o usuaria.

El tercero de los dilemas que aparece con mayor frecuencia (en un $43 \%$ ) es el de denunciar o no a otros profesionales por incompetencia profesional. Muchos de los dilemas de este tipo se presentan en situaciones en las que no se puede calificar el comportamiento del otro profesional como totalmente negligente o muy grave, pero sí como una mala práctica profesional.

En general, hay una cierta resistencia a denunciar a compañeros, aunque tampoco conviene olvidar que las consecuencias de algunas actuaciones profesionales pueden ser graves. En el Código deontológico de trabajo social se habla de los principios de compañerismo, lealtad y respeto recíproco que deben guiar las relaciones con otros profesionales, pero también se afirma (artículo 56) la obligación de comunicar por escrito a la Junta de Gobierno del Colegio el incumplimiento de las normas del código.

La confidencialidad es otro de los principios éticos en torno al cual surgen más dilemas éticos. Las trabajadoras y los trabajadores sociales han de respetar el derecho de los usuarios y las usuarias a «la intimidad, confidencialidad y uso responsable de la información» (artículo 11 del Código deontológico). Todo el capítulo vi del Código deontológico está dedicado al secreto profesional y se concretan aspectos relativos a los sistemas de informatización de los datos, a facilitar información sin datos identificativos de los usuarios, etc. Además, al igual que hemos señalado respecto a la autonomía, el respeto a la confidencialidad y el secreto profesional también tiene excepciones y limitaciones, como, por ejemplo, el trabajo en equipo dentro de la intervención profesional, el perjuicio al propio usuario, el daño a terceras personas o al profesional.

Como hemos visto, los dilemas éticos se agrupan en cuatro factores: la información y su tratamiento, los factores intrínsecos a la intervención, los factores extrínsecos a la intervención profesional y los dilemas en torno a las relaciones entre el trabajador o la trabajadora social y los usuarios.

El factor relativo a la información agrupa los dilemas relacionados con el manejo de datos relevantes, la revelación o no de datos de la historia social y datos personales, el deber o no de informar a terceras personas, la confidencia- 
lidad, el modo de realizar informes, decir o no toda la verdad y, finalmente, el modo de obtener el consentimiento informado por parte de los usuarios. Hemos llamado «reveladores» al tipo de profesionales entre los que se dan de forma mayoritaria este tipo de dilemas, y en este grupo se sitúa casi el 20\% de los profesionales encuestados.

Los factores intrínsecos a la intervención incluyen aspectos como: duración del tiempo de intervención, distribución de recursos disponibles, contraprestaciones económicas o materiales, conflicto de intereses, abuso de poder $\mathrm{y}$ respeto a la autonomía del usuario. Este tipo de dilemas sucede de forma mayoritaria entre los profesionales a los que hemos llamado «esenciales» y agrupan al 17,8\% del total de encuestados.

El tercer grupo de dilemas éticos se refiere a lo que hemos llamado «factores extrínsecos a la intervención». Incluye temas relativos a la información que se da a los medios de comunicación, la incompetencia de otros profesionales, la asistencia a juicios y la responsabilidad por actuaciones que han perjudicado a un colega. Estos dilemas suceden mayoritariamente entre los profesionales a los que hemos llamado "externos", que representan el 26,5\% del total de encuestados.

Finalmente, el cuarto factor agrupa los dilemas vinculados al tipo de relación que se establece entre trabajadores sociales y usuarios. En ocasiones, este tipo de relación va más allá de los límites estrictamente profesionales. Estos dilemas tienen lugar entre los profesionales a los que hemos llamado "compañeros» y agrupan al 24,4\% del total de encuestados.

\section{Agradecimientos}

En este artículo, se exponen parte de los resultados del proyecto de investigación Dilemas éticos en la intervención social: La perspectiva de los trabajadores sociales en España (FFI2008-05546), financiado por el Plan Nacional de I+D+i del Ministerio de Ciencia e Innovación (2008-2010).

Agradecemos su colaboración al Consejo General de Colegios Oficiales de Diplomados en Trabajo Social y Asistentes Sociales de España en el envío de los cuestionarios. También agradecemos, de un modo muy especial, la colaboración de todas las trabajadoras y los trabajadores sociales españoles que han dedicado parte de su tiempo a contestar y enviar de forma anónima el cuestionario utilizado en esta investigación. Sin sus aportaciones, este trabajo no hubiera sido posible. 


\section{Referencias bibliográficas}

Ain, E.J. (2003). Ethical Dilemmas of New York City Social Workers. Michigan: Ph. D. Ann Arbor.

Ballestero, A. (2006). Dilemas éticos en Trabajo Social. Pamplona: Eunate.

BAnks, S. (2001). Ethics and Values in Social Work. Londres: McMillan Press.

- (2004). Ethics, Accountability and the Social Professions. Houndmills, Basingstoke, Hampshire: Palgrave MacMillan.

- (2005). «Accounting for Ethical Difficulties in Social Welfare Work». British Journal of Social Work, 35, 1005-1022.

Bermejo, F.J. (2002). La ética del trabajo social. Bilbao: Desclée de Brouwer.

Chomsky, N. (1974). Estructuras sintácticas. Madrid: Siglo XXI.

Colegio Oficial de Diplomados en Trabajo Social y Asistentes Sociales (1999). Código deontológico de trabajo social en España, aprobado por la Asamblea General de Colegios Oficiales de Diplomados en Trabajo Social y Asistentes Sociales de 29 de mayo de 1999.

Congress, E.P. (1999). Social Work Values and Ethics: Identifying and Resolving Professional Dilemmas. Belmont: Wadsworth Group / Thomson Learning.

«Ley Orgánica 15/1999, de 13 de diciembre, de protección de datos de carácter personal». Boletín Oficial del Estado, 298, 14 de diciembre de 1999, 43088-43099.

Linzer, N. (1999). Resolving Ethical Dilemmas in Social Work Practice. Boston, MA: Allyn \& Bacon.

Linzer, N.; Conboy, A. y Ain, E. (2003). «Ethical Dilemmas of Israeli Social Workers». International Social Work, 46 (1), 7-21.

Loewenberg, F.M. y Dolgoff, R. (1996). Ethical Decisions for Social Work Practice. Itasca, Illinois: F. E. Peacock Publishers, Inc.

Lorenz, W. y Seibel, F. (1999). "European Educational Exchanges in the Social Professions. The ECSPRESS Experience». En: Marynowicz-HetKa, E.; Wagner, A. y Piekarski, J. (eds.). European Dimensions in Training and Practice of the Social Professions. Katowice, Poland: Slask, 315-341.

Martínez Ramos, E. (1984). «Aspectos teóricos del Análisis de Cluster y aplicación a la caracterización del electorado». En: SÁnchez CARrión, J.J. (ed.). Introducción a las técnicas de análisis multivariable. CIS: Madrid, 165-208.

Mcauliffe, D. (2000). Beyond the Hypothetical: Practitioner Experiences of Ethical Dilemmas in Front-Line Social Work. Brisbane: University of Queensland. Tesis no publicada.

- (2005). «I'm Still Standing: Impacts \& Consequences of Ethical Dilemmas for Social Workers in Direct Practice». Journal of Social Work Values and Ethics [en línea], primavera, vol. 2 (1). < http://www.socialworker.com/jswve/>.

National Association of Social Workers (NASW) (1998). Carol Brill, Ph.D., NASW FOCUS [en línea]. Hoja informativa, abril. <http://www.naswma.org/>.

- (1999). Code of Ethics of the National Association of Social Workers [en línea]. <http://www.socialworkers.org/>.

Perlman, H.H. (1980). El trabajo social individualizado. Madrid: Rialp.

Preston, N. (2001). Understanding Ethics. Sydney: Federation Press.

Reamer, F.G. (1983). «Ethical Dilemmas in Social Work Practice». Social Work, enero-febrero, 28 (1), 31-35.

- (1990). Ethical Dilemmas in Social Service: Guide for Social Workers. Nueva York: Columbia University Press.

Rhodes, M.L. (1991). Ethical Dilemmas in Social Work Practice. Milwaukee, Wisconsin: Routledge. 
Rothman, J. (1998). From the Front Lines. Student Cases in Social Work Ethics. Boston: Allyn and Bacon.

Salcedo, D. (1998). Autonomía y bienestar: La ética del trabajo social. Granada: Comares.

Strom-Gottrried, K. (2006). Ethics in Social Work Practice: A Primer to Accompany. Nueva York: McGraw-Hill Companies.

Úriz, M.J. y Ballestero, A. (2006). "Ethical dilemmas of social workers in the social services: The case of Navarre (Spain)». European Journal of Social Education / Journal Europeen d'Education Social, 10-11, 133-145.

Úriz, M.J.; Ballestero, A. y Urien, B. (2007). Dilemas éticos en la intervención social: Una perspectiva profesional desde el trabajo social. Zaragoza: Mira. 